\title{
Hypoxia induced interstitial transformation of microvascular endothelial cells through mediating HIF-1a/VEGF signaling in systemic scleroderma
}

\section{Jing Mao}

the affilliated hospital of southwest medical university

Jiexiong Liu

The affilliated hospital of southwest medical university

Mei Zhou

The southwest medical university

\section{Guiqiang Wang}

Peking University First Hospital

\section{Xia Xiong}

The Affilliated Hospital of Southwest Medical University

\section{Yongqiong Deng ( $\square$ dengyongqiong1@126.com )}

The Affiliated Hospital of Southwest Medical University https://orcid.org/0000-0003-2023-830X

\section{Research article}

Keywords: Hypoxia, scleroderma, endothelial interstitial transformation, Hypoxia inducible factor-1a, vascular endothelial growth factor a

Posted Date: March 28th, 2020

DOl: https://doi.org/10.21203/rs.3.rs-19468/v1

License: (c) (i) This work is licensed under a Creative Commons Attribution 4.0 International License. Read Full License 


\section{Abstract}

Background: Endothelial mesenchymal transition (EndMT) is a key pathological event for vasculopathy which is one of the early features and hallmarks of systemic scleroderma (SSc). It is well established that hypoxia contributes to EndMT, however, little is known about the effects of EndMT induced by hypoxia on skin microvascular remodeling of SSc,as well as the underlying mechanism.

Methods: Skin biopsy was down for SSc patients and healthy controls and skin tissues were collected for iTRAQ-based proteomics and Immunohistochemical test. Human microvascular endothelial cell line (HMEC-1) cultured with hypoxic or normal conditions, treated by either tamoxifen or bevacizumab. The expression of Hypoxia inducible factor-1a (HIF-1a), vascular endothelial growth factor (VEGF)-a, CD31, asmooth muscle actin (a-SMA), VE-cadherin, fibronectin were detected at both protein and mRNA level.

Results : The iTRAQ-based proteomics indicated significantly up-regulated HIF-1 sigaling in the skin tissues of SSc patients. Immunohistochemical results demonstrated a significant downregulation of the endothelial cell (EC) marker CD31 and a distinct positive staining of the interstitial cell (IC) marker a-SMA at sites lining the vessel lumens in the skin tissues of SSc. Meanwhile the positive staining of HIF-1 $\mathrm{a}$, a key transcription factor in response to chronic hypoxia, and VEGF-a were found to be diffusely distributed in SSc skin tissue. Consistent with these observations, HMEC-1 cells cultured under hypoxic conditions exhibited a significant decrease in CD31 and VE-cadherin expression, alongside a marked increase in the expression of a-SMA and fibronectin, as well as a distinct up-regulation of HIF-1a and VEGF-a, while comparing with them under normal condition. Of note, the inhibition of HIF-1a by tamoxifen effectively down-regulated the hypoxic induction VEGF-a, a-SMA, while rescuing hypoxic suppression of CD31. In addition, a VEGF-a inhibitor bevacizumab treatment had the same effect on the hypoxic expression of aSMA and CD31 as tamoxifen intervention, but did not reduce HIF-1a.

Conclusion: These results suggest that the HIF-1a/VEGF signaling pathway has a critical role in mediating the effect of hypoxia-induced EndMT on skin microvascular remodeling of SSc.

\section{Background}

Systemic sclerosis is a rare connective tissue disease with high disability and mortality rates, featured by autoimmunity, a broad microvasculopathy, and fibrosis of the skin and of visceral organs[1]. EndMT has emerged as a key player in the pathogenesis of tissue fibrosis and fibroproliferative vasculopathy in various diseases, including cardiac and intestinal fibrosis, diabetic nephropathy, portal hypertension, and pulmonary arterial hypertension (PAH) [1, 2]. As well, experimental evidence supported a role of EndMT in lung tissues of patients with interstitial lung disease associated with $\mathrm{SSc}[3,4]$. Additionally, cells in the intermediate stages of EndMT were identified in dermal microvessels of both patients with SSc and bleomycin-induced and urokinase-type plasminogen activator receptor deficient mouse models[5]. That is, EndMT causally connect two hallmarks of SSc, the persistent endothelial injury and the aberrant fibrogenesis. 
In the pathogenesis of SSc, reduced capillary blood flow coupled with deficient angiogenesis leads to chronic hypoxia, enforcing a positive feed-forward loop sustaining vascular remodelling, which further promote the irreversible extensive tissue fibrosis[2]. It indicates that chronic hypoxia is a prominent trait contributing to vasculopathy and tissue fibrosis in SSc. In recent years, a large number of studies have confirmed that hypoxia could induce EndMT in vitro and in vivo[6, 7, 8]. However, the process of EndMT induced by hypoxia in the pathogenesis of SSc was not clear.

HIF-1a is a key transcription factor in response to chronic hypoxia and is currently considered to be the "main regulatory factor" of hypoxia environment[9]. It was reported that HIF-1 a promoted fibrotic disease and its implicated function included stimulation of excessive ECM, vascular remodeling, and futile angiogenesis with further exacerbation of chronic hypoxia and deteriorate pathofibrogenesis, as well as EndMT[10,11]. Excessive expressions of HIF-1a has been detected in the tissues of systemic sclerosis[12, 13]. In addition, VEGF is the predominant proangiogenic factor regulated by HIF-1a in hypoxia related diseases, and remarkable up-regulation of VEGF has been observed in SSc specimen[10, 13]. Recently, the inhibition of HIF-1a/VEGF signaling pathway was reported to reverse the anti-angiogenesis effects of chrysophanol[14]. Nevertheless, whether hypoxia induced EndMT in the pathogenesis of SSc depended on HIF-1a/VEGF signaling needs to be further verified.

Herein, we provided immunohistological evidence verifying the EndMT in the dermal specimens of patients with SSc, as well as the excessive increased expression of HIF-1a and VEGF-a. We further show that HMEC-1 cells cultured under hypoxic conditions exhibited the characteristics of EndMT and the activation of both HIF-1a and VEGF-a. Finally, the inhibition of HIF-1a/VEGF signaling by tamoxifen and bevacizumab effectively inhibited EndMT. These observations provide strong support to the hypothesis that the HIF-1a/VEGF signaling has a critical role in mediating the effect of hypoxia-induced EndMT on skin microvascular remodeling of SSc. This novel mechanism may represent an important and novel therapeutic target for the complications of SSc-associated fibroproliferative vasculopathy and fibrosis.

\section{Materials And Methods}

\section{Patients and tissue samples}

Skin tissue samples from 8 patients with SSc and 8 age and gender matched healthy controls were studied. Tissue samples of patients and controls were obtained by skin biopsy performed at the Affiliated Hospital of Southwest Medical University. We included only those patients who met the American College of Rheumatology (ACR)/European League Against Rheumatism(EULAR) 2013 classification criteria for SSc [15]. Eligibility criteria excluded the following key conditions: (1) Aged < 18 or $>75$ years. (2)BMI>25. (3)Patients had severe primary diseases such as liver, kidney, hematopoietic system damage, and other skin and connective diseases. (4) Patients with recent infection. (5) Tumor patients. The control samples were obtained from the normal skin surrounding nevus, when it was removed. All the specimens were taken from the fingers, back of the hand and forearm. All the surgical biopsies were performed following 
the patients' provision of informed consent and according to Institutional Review Board-approved protocols from the Affiliated Hospital of Southwest Medical University.

\section{Histopathology, immunohistochemistry}

The qualified skin tissue was fixed with 10\% formalin, embedded in paraffin and sliced at $4 \mu \mathrm{m}$. Slides containing paraffin-embedded skin tissue sections were examined histopathologically by staining with hematoxylin and eosin, and also by immunohistochemical staining with specific EC and mesenchymal (myofibroblast) cell markers. The procedure of immunohistochemical staining was as following. After dewaxing and hydration, the specimens were washed with PBS and repaired with antigen in citric acid buffer. Catalase blockers were added, and the antibodies were added in turn. Slides were first incubated overnight with one of the following antibodies: anti-CD31 (1:50 dilution), anti-a-SMA (1:200 dilution), antiHIF-1a (1:100 dilution), and anti-VEGF-a (1:100 dilution). IgG binding was revealed following incubation with secondary antibodies for 1 hour. Then the slides were colored with DAB chromogenic agent. Finally, the positive signals of each index were semi-quantitatively counted by Image $\mathrm{J}$, and the analysis was carried out.

\section{Extraction of total proteins from skin tissues}

The skin tissues were taken from 4 patients and matched controls, immediately soaked in liquid nitrogen before storing them at $-80^{\circ} \mathrm{C}$. Each tissue was ground in lysis buffer using Tissue Lyser II at a frequency of $25 \mathrm{~Hz}$ for a period of $30 \mathrm{~min}$. The skin tissues were chopped up with scissors, then additional $30 \mathrm{~min}$ of sonication were added using the intelligent ultrasonic processor DH99-IIIN (lawson, China) to lyse well the tissues until a total grinding. Then, tissues were lysed in a lysis buffer ( $4 \%$ SDS, 0.1M DTT in $0.1 \mathrm{M}$ Tris- $\mathrm{HCl}, \mathrm{pH} 7.6)$ at a ratio of $100 \mathrm{mg}$ tissue per $1 \mathrm{~mL}$ lysis buffer. The tissues were vortexed and incubated for $5 \mathrm{~min}$ at $95^{\circ} \mathrm{C}$ before being lysed for $20 \mathrm{~s}$ with $1 \mathrm{sec}$ OFF $1 \mathrm{sec}$ ON at $20 \%$ amplitude using ultrasonic processor probe. Then, the lysate was centrifuged at $14,000 \times \mathrm{g}$ for $20 \mathrm{~min}$ at $4{ }^{\circ} \mathrm{C}$ to get rid of the cell debris. The supernatant was recovered in a new tube and the amount of proteins was measured using BCA Protein Assay Kit according to the manufacture's instruction. The protein assay by the BCA method is used for the quantification of total proteins in a sample.

\section{Absolute quantitation (iTRAQ)-based proteomics}

An amount of $1 \mathrm{mg}$ of protein from each sample was deposited in a filtration centricon Microcon YM-30 (Millipore). Washing steps were performed with $8 \mathrm{M}$ urea, $0.1 \mathrm{M}$ Tris-HCl, $\mathrm{pH} 8.5$ buffer. The cysteine residues were blocked with $12 \mathrm{mM}$ methyl methanethiosulfonate for $30 \mathrm{~min}$ at room temperature. Next, the proteins were digested by trypsin with enzyme to substrate ratio of $1: 50(\mathrm{w} / \mathrm{w})$ at $37^{\circ} \mathrm{C}$ for $15 \mathrm{~h}$. Furthermore, the digested peptides were labeled with iTRAQ Reagent Kit (Applied Biosystems, Foster city, CA, USA). The iTRAQ-labeled samples were analyzed by a NanoAquity UPLC system connected to Q Exactive hybrid quadrupole-Orbitrap mass spectrometer (Thermo Fisher Scientific, USA). All the protein analysis were performed by Majorbio Bio-pharm Technology Co., Ltd (Shanghai, China). The amino acid sequences of identified proteins from iTRAQ were matched to the local protein 
database(https://www.ncbi.nlm.nih.gov/genbank/), and KEGG (http://www.genome.jp/kegg/) databases to search protein candidates and associated pathways.

\section{Reagents and antibodies}

For immunohistochemistry and western blot, the following primary antibodies were used: anti-CD31, antia-SMA, anti-HIF-1 $a$, anti-VEGF-a. They were all purchased from Abcam (USA). The mouse Anti-Rabbit IgG (1:5000 dilution) was purchased from Santa Cruz (USA). The anti-b-actin and Goat Anti-Mouse IgG (1:3000 dilution) were from Bioss (China). The specific HIF-1a inhibitor tamoxifen was purchased from Solarbio (China). The specific VEGF-a inhibitor bevacizumab was purchased from MedChemExpress (USA).

\section{Cell culture}

HMEC-1 cell line was obtained from Zhongqiao Xinzhou Company (Shanghai, China). The cells were cultured in sciencell brand ECM medium containing $5 \%$ fetal bovine serum(FBS), $1 \% \mathrm{P} / \mathrm{S}$ double antibody and $1 \%$ ECGS endothelial growth factor. Cells were maintained at $37^{\circ} \mathrm{C}$ in a humidified $5 \% \mathrm{CO} 2$ in air atmosphere. The anaerobic gas production bag (Hpebio, Qingdao, China) was used to promote hypoxia environment, by putting it into the anaerobic culture box. Then the HMEC-1 cells were treated with hypoxia by culturing in the anaerobic culture box for $72 \mathrm{~h}$. Phenol blue oxygen indicator was used to monitor oxygen concentration and hypoxia status.

Tamoxifen was dissolved in DMSO to construct a solution of $5 \mathrm{mmol} / \mathrm{L}$ concentration, then filtered through a needle sterile filter and stored at $-20^{\circ} \mathrm{C}$ in dark. The HMEC- 1 cells also coule be co-cultured with 5 umol/L tamoxifen, and with DMSO as the negative control. Bevacizumab was dissolved in PBS to construct a solution of $3 \mathrm{mg} / \mathrm{ml}$ concentration, then filtered through a needle sterile filter and stored at -20 ${ }^{\circ} \mathrm{C}$ in dark. The HMEC- 1 cells also could be co-cultured with $0.3 \mathrm{mg} / \mathrm{ml}$ bevacizumab, and with PBS as the negative control.

\section{Quantitative reverse transcription-polymerase chain reaction (PCR)}

Total RNA was extracted using TRIzol reagent (Invitrogen, Thermo Fisher Scientific, USA), according to the manufacturer's instructions. And $\beta$-actin was used as internal control to detect the mRNA expression of transcription factors HIF-1a, VE-cadherin, Fibronectin, CD31, a-SMA, VEGF-a, VEGFR1 and VEGFR2. The EC transcript levels were quantified using Rever Tra Ace qPCR RT Kit (Code No.FSQ-201,TOYOBO,Japan) on the ABI PRISM 7500 system (Applied Biosystems, USA). Quantifification was performed using $2^{-\triangle \triangle C T}$ method. The primers used in the PCR analyses are as followings.

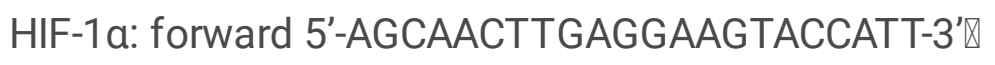

Reverse 5'-AGGTGAACTTTGTCTAGTGCTT-3'

VE-cadherin: forward 5'-AAAGAATCCATTGTGCAAGTCC-3'囚 
Reverse 5'-CGTGTTATCGTGATTATCCGTG-3'

Fibronectin: forward 5'-AATAGATGCAACGATCAGGACA-3'】

Reverse 5'-GCAGGTTTCCTCGATTATCCTT-3'

CD31: forward 5'-TCGTGGTCAACATAACAGAACT-3'】

Reverse 5'-TTGAGTCTGTGACACAATCGTA-3'

a-SMA: forward 5'-CCGGGAGAAAATGACTCAAATT-3'】

Reverse 5'-CTCAGCAGTAGTAACGAAGGAA-3'

VEGF-a: forward 5'-ATCGAGTACATCTTCAAGCCAT-3'】

Reverse 5'-GTGAGGTTTGATCCGCATAATC-3'

VEGFR1: forward 5'-CAAGATTTGCAGAACTTGTGGA-3'】

Reverse 5'-CTGTCAGTATGGCATTGATTGG-3'

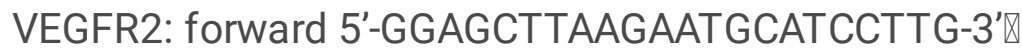

Reverse 5'-GATGCTTTCCCCAATACTTGTC-3'

\section{Western blot}

HMEC-1 cells were lysed with RIPA lytic buffer (Beyotime Biotechnology,China). The same amount of protein samples were separated by $10 \%$ SDS-PAGE and transferred to NC membrane (Milliporea, USA). After $5 \%$ skim milk was blocked, the membranes were incubated overnight at $4{ }^{\circ} \mathrm{C}$ with one of the following primary antibody, $\beta$-actin (1:1000), HIF-1a (1:1000), a-SMA (1:500), CD31 (1:500), VEGF-a (1:500). Then, the membranes were washed with PBS and incubated for 1 hour with the secondary antibodies. Finally, they were visualized by ECL reagent (Millipore, USA). Protein images were captured using Image $\mathrm{J}$.

\section{Statistical analysis}

All experiments were repeated at least three times with the similar results. The values were expressed as mean \pm SD of at least three independent experiments in triplicate. Statistical differences were assessed by a one-way analysis of variance (ANOVA) (Kruskal-Wallis) in SPSS17.0 software. A P value of less than 0.05 was considered statistically significant.

\section{Results}


The clinical features of the SSc patients and healthy controls whose skin tissues were studied were shown in Table 1. Protein patterns from 4 patients and 4 controls were analyzed by iTRAQ-based quantitative approach. A total of 2159 proteins were identified. The amino acid sequences of identified proteins the protein dataset was matched to the local database, and 43 proteins were found to be significantly different between SSc patients and controls with $P$ value $<0.05, F C>1.2$ or $<0.83$, shown in Additional file 1. Functional enrichment analysis was performed for the identified proteins based on KEGG databases, the top 15 up and down pathways were shown in fig. 1A. Interestingly, the HIF-1sigaling significantly up-regulated in the skin tissues of SSc patients.

Table 1. Clinical and demographic characteristics of SSc patients and controls.

\begin{tabular}{llll}
\hline Characteristics & SSc $(\mathrm{n}=8)$ & Controls $(\mathrm{n}=8)$ & P value \\
\hline Age(years) & $51 \pm 8.49$ & $51 \pm 8.49$ & 1.00 \\
Female/male & $6 / 2$ & $6 / 2$ & 1.00 \\
$\mathrm{BMI}\left(\mathrm{Kg} / \mathrm{m}^{2}\right)$ & $21.75 \pm 2.63$ & $22.06 \pm 1.19$ & 0.77 \\
Duration of disease (years) & $1.04 \pm 0.82$ & - & - \\
Involve organs (n/N) & & & - \\
Raynaud phenomenon & $7 / 8$ & - & - \\
Lung & $4 / 8$ & - & - \\
Gastrointestinal tract & $3 / 8$ & - & - \\
Joint & $5 / 8$ & & - \\
Kidney & $1 / 8$ & - & 1.00 \\
Heart & $2 / 8$ & - & \\
Biopsy site (n) & & & \\
Finger/Back of hand/ Forearm & $4 / 2 / 2$ & $4 / 2 / 2$ & \\
& & & \\
\hline
\end{tabular}

\section{EndMT and activated HIF-1a/VEGF signal were detected in the patients with systemic scleroderma}

Compared with controls, all 8 skin tissue specimen from the SSc patients with displayed varying degrees of interstitial fibrosis, along with a pattern of infiltration of inflammatory mononuclear cells around vessels, marked narrowing, occlusion of the vessel lumen and vascular loss, as illustrated in Fig. 1B, C. The EC marker CD31 and IC marker a-SMA were used in immunohistologic analyses to identify EndMT present in the cutaneous tissue. As expected, CD31 positive cells significantly decreased in the SSc tissue were detected at sites lining the vessel lumens, compared with them in control tissue, as illustrated in Fig.1D, E. Meanwhile, positive a-SMA staining of a-SMA was shown perivascularly in specimens of patients and on the epidermal cells, which was negative in control specimen, as illustrated in Fig.1F,G.

The HIF-1a/VEGF signal markers were also analyzed by immunohistochemistry. All skin biopsies from SSc patients were both HIF-1 $a$ and VEGF positive, however HIF-1 $a$ was not present and VEGF expressed lowly in the healthy tissue samples (Fig. $1 \mathrm{H}, \mathrm{I}, \mathrm{J}, \mathrm{K}$ ). The staining of the two markers were observed throughout the keratinocytes of the epidermis, as well as diffusely distributed in dermis.

Hypoxia induced interstitial transformation of microvascular endothelial cells in vitro 
To test whether hypoxia is able to induce the conversion of ECs into ICs, EC cell line HMEC-1 were exposed to anaerobic gas production bag for hypoxia treatment and endothelial and fibrotic characteristics were measured. ECs exposed to anaerobic gas exhibited a decrease for the mRNA expression of the endothelial proteins VE-cadherin and CD31 (Fig. 2Aand B) at every hypoxia time point that was tested. Furthermore, consistent with the establishment of a fibrotic process, ECs challenged with anaerobic gas showed an increase for the mRNA level in the fibrotic markers a-SMA and fibronectin (Fig. $2 \mathrm{C}$ and $\mathrm{D}$ ) at every hypoxia time point that was tested. At protein level, the expression level of CD31 was up-regulated temporarily after 24 hours of hypoxia, and then declined gradually with the extension of hypoxia. The protein of a-SMA showed an continuous increase with the prolongation of hypoxia treatment, as illustrated in Fig. 2 E,F,G.

We also investigated the effect of hypoxia on the cellular morphology and polarity of ECs. In the absence of hypoxia, HMEC-1 cells were circular adherent cells. When the cells were anoxic for 12 hours, there was a small amount of death, but the shape was still full and the outline is clear. With the duration of hypoxia, the number of cell death increased, and the morphology changed to fusiform, losing adhesion connection and polarization gradually. The data was shown in Additional file2.

\section{Hypoxia activated the HIF-1a and VEGF signaling in vitro}

Considering the postulation of HIF-1a/VEGF signaling pathway involving in hypoxia-mediated EndMT, we were prompted to investigate whether hypoxia induced the activation of signal molecules and their ligands at both mRNA and protein levels. There was no change of HIF-1 a mRNA level at different time points after hypoxia (Fig. 3 A). But ECs in the presence of hypoxia exhibited increased VEGF-a mRNA level at every time point that was tested(Fig. 3 B). Meanwhile, the expression of two ligands of VEGF, VEGF-R1 and VEGFR2 were detected at different time points of hypoxia by qRT-PCR. To that end, the activation of VEGF-R1 and the inhibition of VEGF-R2 were found, as illustrated in Fig. $3 \mathrm{C}$ and D. At protein level, the detection of HIF-1 $a$ and VEGF-a were performed by western blot, which indicated the up-regulation of both molecules(Fig. 3 E,F,G).

\section{Down-regulation of HIF-1a inhibits hypoxia induced EndMT and VEGF Signaling}

Although tamoxifen has been reported to be the specific inhibitor of HIF-1a[16], whether HIF-1a signaling and its probable downstream VEGF signaling induced by hypoxia could be effectively abolished needed further investigation. To test this, hypoxia-treated ECs incubated with tamoxifen for 72 hours and the signal molecules HIF-1 $\mathrm{a}$ and VEGF-a were detected at protein level. Results showed that hypoxia treated ECs with tamoxifen showed a sustaining decreased expression of both HIF-1a and VEGF-a(Fig. 4 A,B,C).

Taking into account that hypoxia induces EndMT and the expression of HIF-1a and VEGF-a, we tested whether the activation of the HIF-1a/ VEGF signaling is necessary for the EndMT induced by hypoxia. To that end, we used tamoxifen the specific inhibitor of HIF-1a/ VEGF signaling and tested the expression of CD31, a-SMA and fibronectin in hypoxia treated EC. As a result, the decreased mRNA expression of EC markers CD31 in hypoxia treated HMEC-1 cells could be inhibited by tamoxifen intervention(Fig. 5 A). 
Moreover, the hypoxia treated EC with tamoxifen showed a significant decrease in the mRNA expression of fibrotic protein a-SMA and fibronectin, which could up-regulate in EC induced by hypoxia without the inhibition of HIF-1a/ VEGF signaling (Fig. 5 B,C). The protein levels of CD31 and a-SMA were also detected by Western blot. We found that the expression of EC marker CD31 could not decrease and even the IC marker a-SMA was inhibited in hypoxia treated EC, while co-culturing with tamoxifen (Fig. 5 D,E,F).

\section{Hypoxia induced EndMT is dependent on the activation of HIF-1a/ VEGF signaling}

Although the VEGF-a has been verified as the predominant proangiogenic factor regulated by HIF-1a in hypoxia related diseases, it's unknown whether the hypoxia-induced EndMT mediated by up-regulation of HIF-1a signaling was dependent on the activation of VEGF-a. Then the bevacizumab, the inhibitor of VEGF-a was used to treat the ECs in hypoxia conditions for 72 hours and HIF-1a ,VEGF-a, CD31 and aSMA were detected at protein level[17]. The VEGF-a could be inhibited gradually by its inhibitor which could not intervene the activation of HIF-a induced by hypoxia(Fig.6A, B, C). The down-regulation of CD31 and up-regulation of a-SMA induced by hypoxia could be rescued perfectly by the inhibition of VEGF-a (Fig. 6 D,E,F).

\section{Discussion}

Therapeutic improvements have been reported to alleviate the relative damage of SSc complications on patients' prognosis, however previous articles did highlight a high mortality rate in SSc patients and SSc vasculopathy, in terms of pulmonary PAH, heart involvement, and scleroderma renal crisis, still represents the first cause of disease related mortality [2, 18-21]. In deed, vasculopathy is present not only early but almost invariably during the course of SSc, which is characterized by the frequent perivascular inflammation, intimal hyperplasia, medial thickening, obliteration of the lumen, vascular loss and occasionally microthrombi[22]. The mechanism of SSc vasculopathy is not fully understood, but increasing evidence indicates that endothelial injury and subsequent endothelial dysfunction is a primary event that triggers the subsequent formation of typical vascular lesions[23]. It is known that EndMT, a key player in the remodelling of injured vessels may be reversible, possibly offering novel cues for treatment[2]. Moreover,the main fibrosis orchestrators in SSc are represented by chronically activated myofibroblasts, and EC was demonstrated to be capable of trans-differentiating toward myofibroblasts, which indicated that EndMT plays a role in both vascular remodeling and tissue fibrosis[5]. However, the machanism underlying the transformation was not completely unveiled.

EndMT refers to a trans-differentiation process during which ECs downregulate the expression of its specific EC markers, such as CD31 and VE-cadherin, acquiring a mesenchymal/myofibroblast phenotype, which is characterized by the expression of SMA, type I collagen, et al[24]. Meanwhile, ECs gain a spindleshaped fibroblast-like morphology, disaggregate, and lose polarity during this transition[24]. In recent years, accumulative evidence supported the prominent role of EndMT in the pathogenesis of tissue fibrosis and fibroproliferative vasculopathy in various diseases,such as $\mathrm{PAH}$, diabetic nephropathy, cardiac and intestinal fibrosis $[25,26]$. Increasing data showed that EndMT emerged as an important 
event in SSc as well[4, 25-27]. In particular, it has been proposed that the SSc-related EndMT process have differential pathogenetic roles depending on the type of affected vessels[24,27]. When affecting capillary vessels, EndMT may lead to an increase in the number of perivascular myobroblasts, thereby contributing to tissue fibrosis and a parallel loss of endothelial cells[27]. In this study, the positive a-SMA staining of a-SMA was shown mainly around the dermis vessels in the specimen of SSc patients.

It is well known that SSc is an autoimmune disease characterized by immunological disturbances, vascular remodeling and broad fibrosis, the order of which remains to be fully determined. Hypoxia is potentially caused by a yet to be indentified circuitry involving such three features that typify SSc. Once present, the hypoxia creates a vicious circle of ongoing pathology. It is clear that hypoxia is an important hallmark in SSc patients[28]. Additionally, previous studies have confirmed that hypoxia could induce EndMT in PHA and myocardial remodeling[6, 8, 29, 30], as well as in scleroderma[7]. Galectin-3, HIF1a/Twist1, BMP-7/Smads and TGF 1 1/SMAD signaling pathways proved to be a critical mediator in PAH by regulating EndMT and a critical role in mediating the effect of hypoxia-induced EndMT in pulmonary arterial and cardiovascular remodeling $[6,8,29,30]$. The vessels involved in these diseases are arterioles and small arteries, in which EndMT may have differential pathogenetic roles from it affecting capillary vessels. Although Liu C, et al. have demonstrated that hypoxia increased vimentin and a-SMA but decreased VE-cadherin and CD31 in HUVECs[7], the pathogenesis underlying EndMT induced by hypoxia was not well studied in SSc.

As a result of the hypoxia in skin and other affected tissue in SSc, activation of important hypoxia pathways is plausible. HIF-1a is the master transcriptional regulator of the adaptive response to hypoxia, which is the tightly regulated form of HIF-1, and will quickly hydroxylated and degraded in normoxic conditions by prolyl hydroxylases [31-33]. But in hypoxia conditions, the expression of HIF-1a upregulate dramatically $[34,35]$. The positive staining of HIF-1 a has been detected throughout the keratinocytes of the epidermis in all skin biopsies from scleroderma patients[13], which was also observed in our study. Although HIF-1a was postulated to be dysregulated in various pathologic conditions, including SSc[3638] and HIF-1 a could induce hypoxia-treated EndMT in pulmonary arterial remodeling through regulating Twist1. The role of HIF-1a in hypoxia induced EndMT of SSc has remained to be fully characterized. This study postulated HIF-1a/VEGF signaling playing a critical role in regulating EndMT induced by hypoxia.

The VEGF has been reported to be significantly up-regulated in all stages of fibrosis and dendritic endothelial cells, which was thought to be one of the main transcriptional targets of HIF-1a in hypoxiarelated diseases $[13,39]$. Traditionally, VEGF was identified as a key mediator of angiogenesis which induced differentiation, proliferation, and migration of endothelial cells, consequently contributing to the formation of vessels through both angiogenesis and vascular remodeling[39]. VEGF exerts its biological functions by binding to its receptorsthe tyrosine kinase receptors VEGFR1 and VEGFR2. However, unexpectedly, skin tissues of SSc patients with characterized vessel obstruction and loss demonstrated a strong upregulation of VEGF and its two receptors, with an intenser staining for VEGFR-2 than for VEGFR$1[13,39]$. Moreover, the increase of VEGF, HIF-1a accompanied by the characteristics of EndMT were observed in the skin of SSc patients in both previous articels and in this study. Meanwhile, HMEC-1 
treated by hypoxic exhibited the downregulated level of CD31, VE-cadherin expression, and a marked increase in the expression of a-SMA and fibronectin, alongside the increased expression of HIF-1a,VEGFa. Of note, the hypoxia induced EndMT was effectively reversed by tamoxifen and bevacizumab, the inhibitor of HIF-1a/VEGF pathway.

\section{Conclusion}

Taken together, the results from this study shown here provide evidence that hypoxia is a crucial factor in inducing the conversion of ECs into ICs through a a HIF-1a/VEGF dependent mechanism that consequently promotes the skin microvascular remodeling and fibrosis in SSc. This information will be beneficial for designing novel and improved therapeutic strategies against the complications of SSc associated fibroproliferative vasculopathy and fibrosis.

\section{Abbreviations}

EndMT endothelial mesenchymal transition

SSc systemic scleroderma

ITRAQ isobaric tags for relative and absolute quantification

HMEC-1 Human microvascular endothelial cell line

HIF-1a Hypoxia inducible factor-1a

VEGF vascular endothelial growth factor

a-SMA a-smooth muscle actin

EC endothelial cell

IC interstitial cell

PAH pulmonary arterial hypertension

ECM extracellular matrix

ACR American College of Rheumatology

EULAR European League Against Rheumatism

PBS phosphate buffer saline

DAB Diaminobenzidine 
SDS Sodium dodecyl sulfate

DTT Dithiothreitol

BCA bicinchoninic acid

KEGG Kyoto Encyclopedia of Genes and Genomes

FBS fetal bovine serum

ECGS endothelial growth factor

DMSO dimethyl sulfoxide

ECL electrochemiluminescence

ANOVA Analysis of Variance

VEGFR Vascular Endothelial Growth Factor Receptor

\section{Declarations}

\section{Ethics approval and consent to participate}

All the surgical biopsies were performed following the patients'provision of informed consent and according to Institutional Review Board-approved protocols from the Affiliated Hospital of Southwest Medical University.

Consent for publication: Not applicable

\section{Availability of data and materials}

The iTRAQ-based proteomics data from this study will be made freely available from the link: http://cloud.majorbio.com; The accession number is deng123.

\section{Competing interests}

The authors declare that they have no competing interests.

\section{Funding:}

This work was supported by research grants from Southwest medical university and luzhou science and technology bureau(Grant number:090300021686).

Authors' contributions: J Mao performed the experiments, analyzed the data, and wrote manuscript. JX Liu collected the data, and revised the manuscript. JX Liu, M Zhou and GQ Wang participated in the 
sample and data collection. Xia Xiong helped optimize the research and proofread the paper. YQ Deng designed and optimized the research, wrote and revised manuscript. All authors read and approved the final manuscript.

Acknowledgements: Not applicable

\section{Contributor Information}

Yongqiong Deng: dengyongqiong1@126.com; Xia Xiong: xiongxia789@126.com

\section{References}

[1] Rosa I, Romano E, Fioretto BS, Manetti M. The contribution of mesenchymal transitions to the pathogenesis of systemic sclerosis. Eur J Rheumatol. 2019; 19: 1-8.

[2] Nicolosi PA, Tombetti E, Maugeri N, Rovere-Querini P, Brunelli S, Manfredi AA. Vascular Remodelling and Mesenchymal Transition in Systemic Sclerosis. Stem Cells Int.2016; doi: $10.1155 / 2016 / 4636859$.

[3] Beon M, Harley R A, Wessels A, Wessels A, Silver RM, Ludwicka-Bradley A. Myofibroblast induction and microvascular alteration in scleroderma lung fibrosis. Clin. Exp. Rheumatol. 2004; 22: 733-42.

[4] Mendoza Fabian A, Piera-Velazquez Sonsoles, Farber John L, Feghali-Bostwick Carol, Jiménez Sergio A. Endothelial Cells Expressing Endothelial and Mesenchymal Cell Gene Products in Lung Tissue From Patients With Systemic Sclerosis-Associated Interstitial Lung Disease. Arthritis and Rheumatology. 2015; 68: 210-217.

[5] Manetti M, Romano E, Rosa I, Guiducci S, Bellando-Randone S, De Paulis A, et al. Endothelial-tomesenchymal transition contributes to endothelial dysfunction and dermal fibbrosis in systemic sclerosis. Annals Rheum Dis. 2017; 76: 924-34.

[6] Zhang Bo, Niu Wen, Dong Hai-Ying, Liu Man-Ling, Luo Ying, Li Zhi-Chao. Hypoxia induces endotheliamesenchymal transition in pulmonary vascular remodeling. Int. J. Mol. Med, 2018; 42: 270-278.

[7] Liu Chaofan, Zhou Xing, Lu Jinghao, Zhu Lubing, Li Ming. Autophagy mediates 2-methoxyestradiolinhibited scleroderma collagen synthesis and endothelial-to-mesenchymal transition induced by hypoxia. Rheumatology (Oxford). 2019; 58: 1966-1975.

[8] Sniegon Isabella, Prieß Mona, Heger Jacqueline, Schulz Rainer, Euler Gerhild. Endothelial Mesenchymal Transition in Hypoxic Microvascular Endothelial Cells and Paracrine Induction of Cardiomyocyte Apoptosis Are Mediated via TGF $\beta_{1} /$ SMAD Signaling. Int J Mol Sci. 2017; doi:10.3390/ijms18112290. 
[9] Lu Xin, Kang Yibin. Hypoxia and hypoxia-inducible factors: master regulators of metastasis. Clin. Cancer Res. 2010; 16: 5928-35.

[10] Xiong Anji, Liu Yi. Targeting Hypoxia Inducible Factors-1a As a Novel Therapy in Fibrosis. Front Pharmacol. 2017; 8: 326.

[11] Xu Xingbo, Tan Xiaoying, Tampe Björn, Sanchez Elisa, Zeisberg Michael, Zeisberg Elisabeth M. Snail Is a Direct Target of Hypoxia-inducible Factor 1a (HIF1a) in Hypoxia-induced Endothelial to Mesenchymal Transition of Human Coronary Endothelial Cells. J. Biol. Chem. 2015; 290: 16653-64.

[12] Deng Wei, Feng Xuebing, Li Xia, Wang Dandan, Sun Lingyun. Hypoxia-inducible factor 1 in autoimmune diseases. Cell. Immunol. 2016; 303: 7-15.

[13] Ioannou Maria, Pyrpasopoulou Athina, Simos Georgios, Paraskeva Efrosyni, Nikolaidou Christina, Venizelos loannis, et al. Upregulation of VEGF expression is associated with accumulation of HIF-1a in the skin of naïve scleroderma patients. Mod Rheumatol. 2013; 23: 1245-8.

[14] Zhang Jie, Wang Qian, Wang Qiang, Guo Peng, Wang Yong, Xing Yuqing, et al. Chrysophanol exhibits anti-cancer activities in lung cancer cell through regulating ROS/HIF-1a/VEGF signaling pathway. Naunyn Schmiedebergs Arch. Pharmacol. 2020; 393: 469-480.

[15] van den Hoogen Frank, Khanna Dinesh, Fransen Jaap, Johnson Sindhu R, Baron Murray, Tyndall Alan, et al. 2013 classification criteria for systemic sclerosis: an American college of rheumatology/European league against rheumatism collaborative initiative. Ann. Rheum. Dis. 2013; 72: 1747-55.

[16] Cortes Ernesto, Lachowski Dariusz, Robinson Benjamin, Sarper Muge, Teppo Jaakko S, Thorpe Stephen D, et al. Tamoxifen mechanically reprograms the tumor microenvironment via HIF-1A and reduces cancer cell survival. EMBO Rep. 2019; doi:10.15252/embr.201846557.

[17] Bahrami Bobak, Shen Weiyong, Zhu Ling, Zhang Ting, Chang Andrew, Gillies Mark C. Effects of VEGF inhibitors on human retinal pigment epithelium under high glucose and hypoxia. Clin. Experiment. Ophthalmol. 2019; 47: 1074-1081.

[18] Rubio-Rivas Manuel, Royo Cristina, Simeón Carmen Pilar, Corbella Xavier, Fonollosa Vicent. Mortality and survival in systemic sclerosis: systematic review and meta-analysis. Semin. Arthritis Rheum. 2014; 44: 208-219.

[19] M. R. Pokeerbux, J. Giovannelli, L. Dauchet, L.Mouthon, C. Agard, J. C. Lega, et al. Survival and prognosis factors in systemic sclerosis: data of a French multicenter cohort, systematic review, and metaanalysis of the literature. Arthritis Res Ther. 2019; 21: 86.

[20] Shouval R, Furie N, Raanani P, Nagler A, Gafter-Gvili A. Autologous Hematopoietic Stem Cell Transplantation for Systemic Sclerosis: A Systematic Review and Meta-Analysis. Biol. Blood Marrow 
Transplant. 2018; 24: 937-944.

[21] Meunier P, Dequidt L, Barnetche T, Lazaro E, Duffau P, Richez C, et al. Increased risk of mortality in systemic sclerosis-associated digital ulcers: a systematic reviewand meta-analysis. J Eur Acad Dermatol Venereol. 2019; 33: 405-409.

[22] Asano Y, Sato S. Vasculopathy in scleroderma. Semin Immunopathol. 2015; 37: 489-500.

[23] Ota Y, Kuwana M. Endothelial cells and endothelial progenitor cells in the pathogenesis of systemic sclerosis. Eur J Rheumatol. 2019; doi:10.5152/eurjrheum.2019.19158.

[24] Rosa I, Romano E, Fioretto BS, Manetti M1. The contribution of mesenchymal transitions to the pathogenesis of systemic sclerosis. Eur J Rheumatol. 2019; doi:10.5152/eurjrheum.2019.19081.

[25] Kanno Y. The role of fibrinolytic regulators in vascular dysfunction of systemic sclerosis. IntJ Mol Sci 2019; doi:10.3390/ijms20030619.

[26] Thuan DTB, Zayed H, Eid AH, Abou-Saleh H, Nasrallah GK, Mangoni AA, et al. A potential link between oxidative stress and endothelial-to-mesenchymal transition in systemic sclerosis. Front Immunol. 2018; 9: 1985.

[27] Manetti M, Guiducci S, Matucci-Cerinic M. The origin of the myo fibroblast in fibroproliferative vasculopathy: does the endothelial cell steer the pathophysiology of systemic sclerosis? Arthritis Rheum. 2011; 63: 2164-7.

[28] van Hal TW, van Bon L, Radstake TR. A system out of breath: how hypoxia possibly contributes to the pathogenesis of systemic sclerosis. Int J Rheumatol. 2011; 2011:824972.

[29] Li Tangzhiming, Zha Lihuang, Luo Hui, Li Suqi, Zhao Lin, He Jingni, et al. Galectin-3 Mediates Endothelial-to-Mesenchymal Transition in Pulmonary Arterial Hypertension. Aging Dis. 2019; 10: 731-745. [30] Zhang Cong-Cong, Zhang Jing-Jing, Chen Jun-Hao, Wu Yuan-Ling, Huang Dan-Na, Dai Yong-Yue, et al. The effect of Yiqi Wenyang Huoxue Huatan Fang on hypoxia-hypercarbia induced pulmonary hypertension and its mechanism. Zhongguo Ying Yong Sheng Li Xue Za Zhi. 2018; 34: 408-413.

[31] Bruick RK, McKnight SL. A conserved family of prolyl-4-hydroxylases that modify HIF. Science. 2001; 294: $1337-40$.

[32] Ke Q, Costa M. Hypoxia-inducible factor-1 (HIF-1). Mol Pharmacol. 2006; 70: 1469-1480.

[33] Simon Siao-Pin, Laura-Otilia Damian, Laura Mirela Muntean, Simona Rednic. Acroosteolysis in systemic sclerosis: An insight into hypoxia-related pathogenesis. Exp Ther Med. 2016. 12: 3459-3463. 
[34] Ivan Mircea, Haberberger Thomas, Gervasi David C, Michelson Kristen S, Günzler Volkmar, Kondo Keiichi, et al. Biochemical purification and pharmacological inhibition of a mammalian prolyl hydroxylase acting on hypoxia-inducible factor. Proceedings of the National Academy of Sciences of the United States of America. 2002; 99: 13459-13464.

[35] van Hal T W, van Bon L, Radstake T R D J. A system out of breath: how hypoxia possibly contributes to the pathogenesis of systemic sclerosis. Int J Rheumatol. 2011; 2011: 824972.

[36] Semenza GL. Hypoxia-inducible factors in physiology and medicine. Cell 2012; 148: 399-408.

[37] Lokmic Z, Musyoka J, Hewitson TD, Darby IA. Hypoxia and hypoxia signaling in tissue repair and fibrosis. Int Rev Cell Mol Biol. 2012; 296: 139-85.

[38] Haase VH. Pathophysiological consequences of HIF activation: HIF as a modulator of fibrosis. Ann N Y Acad Sci. 2009; 1177: 57-65.

[39] Distler Oliver, Distler Jörg H W, Scheid Annette, Acker Till, Hirth Astrid, Rethage Janine, et al. Uncontrolled expression of vascular endothelial growth factor and its receptors leads to insufficient skin angiogenesis in patients with systemic sclerosis. Circ. Res. 2004; 95: 109-16.

\section{Figures}



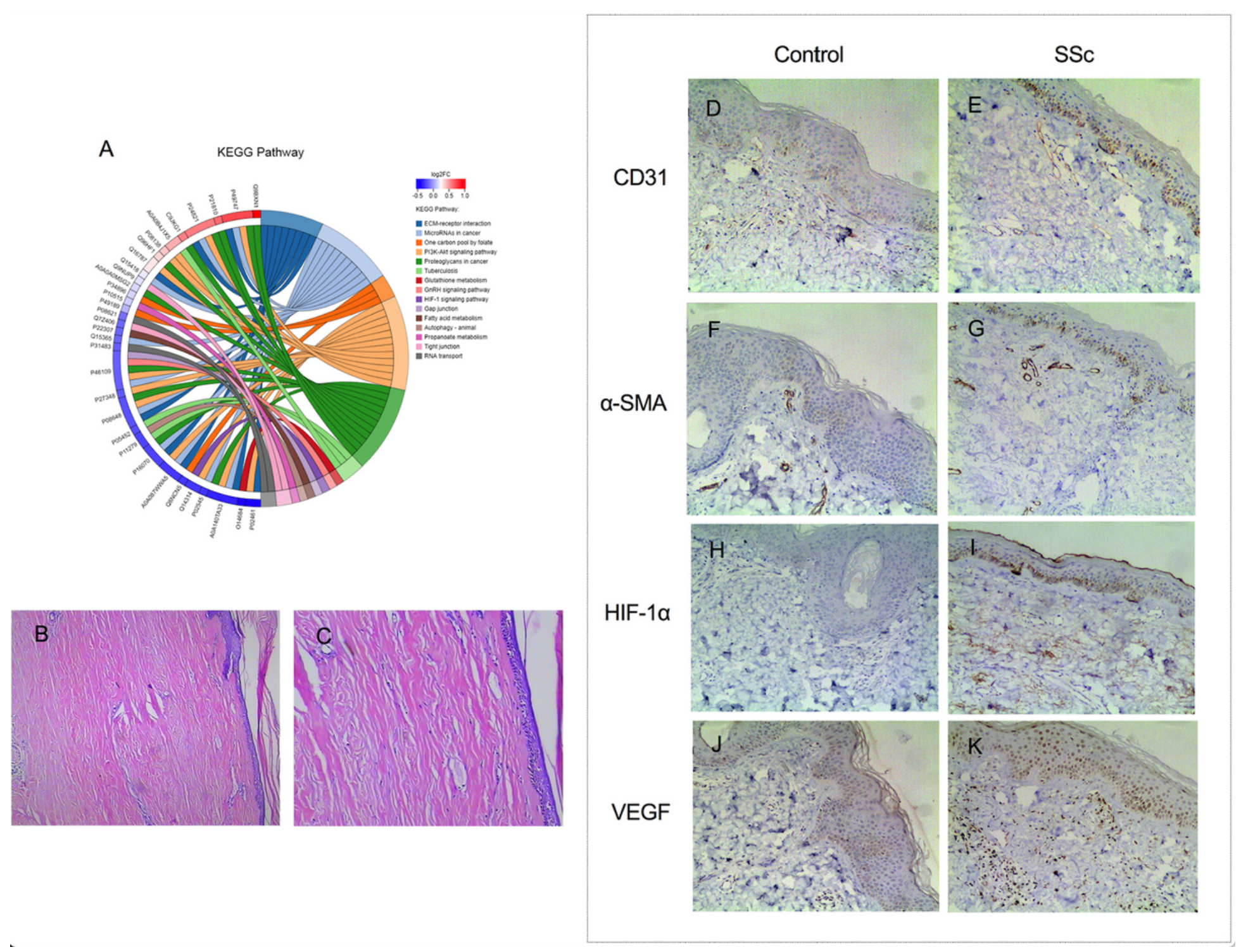

\section{Figure 1}

The iTRAQ-based proteomics, histopathologic and immunohistologic assessments of skin tissue from patients with SSc. Original magnification $\times 20$. (A) The top 15 up and down pathways of functional enrichment analysis performed for the identified proteins based on KEGG databases. (B, C) Hematoxylin and eosin staining of skin tissue of a patient with SSc, indicating a large amounts of fibrous tissue in dermis and infiltration of inflammatory mononuclear cells around vesselsa, as well as narrowing, occlusion of the vessel lumen and vascular loss. (D,E,F,G) Immunohistochemical staining of skin tissue for the EC-specific marker CD31 and IC marker a-SMA. In the tissue from patient as in E, G, the presence of CD31-positive cells decreased and a-SMA-positive signaling increased in the dermis, compared with it in control tissue as in D, F. (H,I,J,k)Immunohistochemical staining of skin tissue for the HIF-1a/VEGF signal. Specimens from SSc a patient were both HIF-1a and VEGF positive as in I,K, and HIF-1a was not present and VEGF expressed lowly in the normal tissue samples as in $\mathrm{H}, \mathrm{J}$. 
A

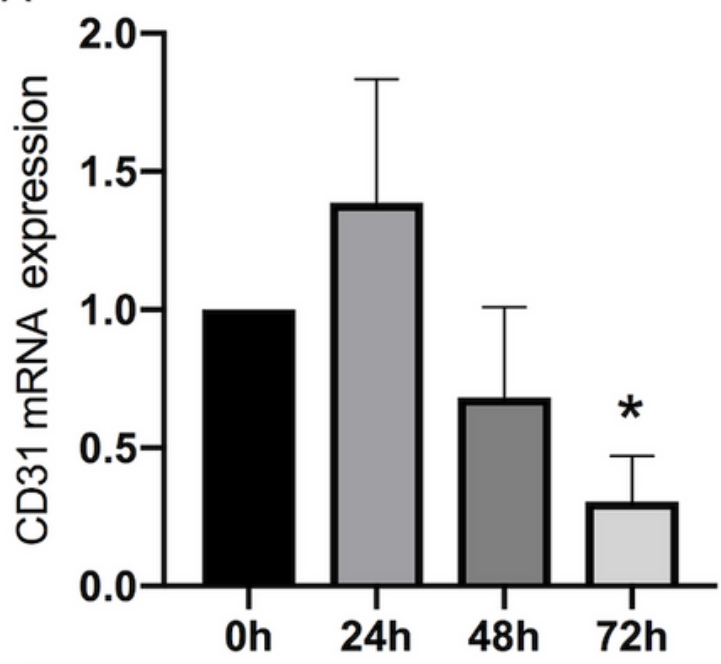

C

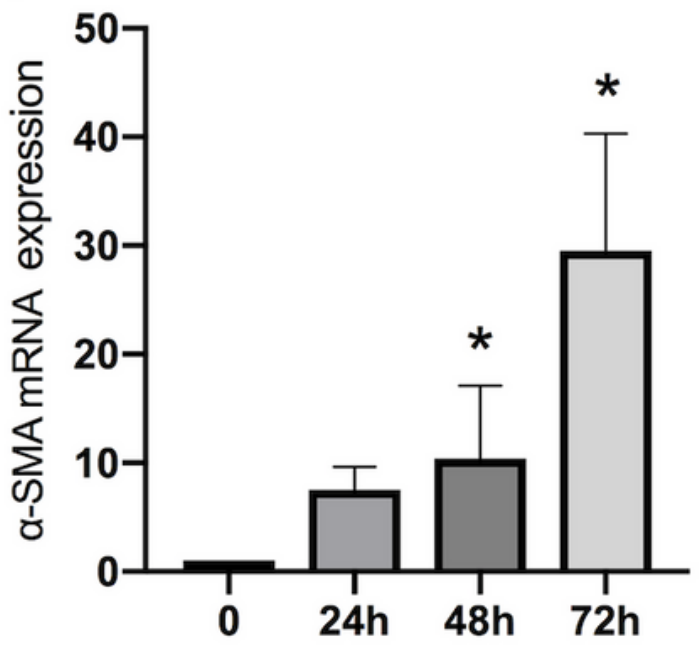

B

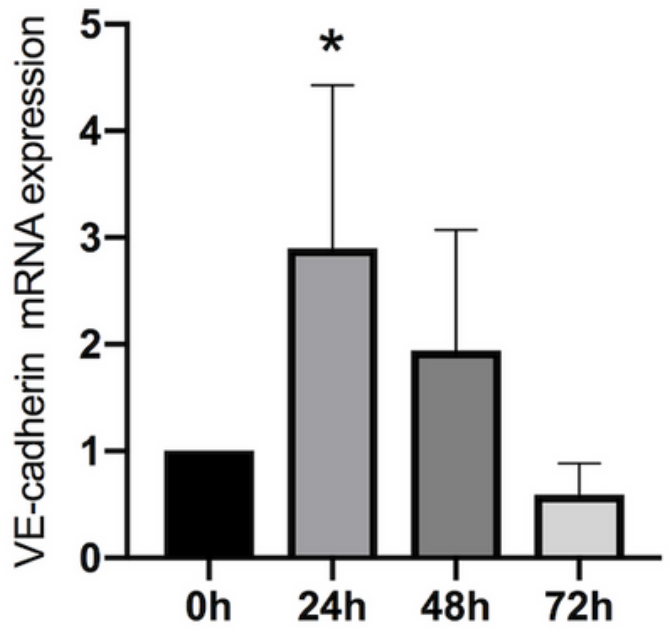

D

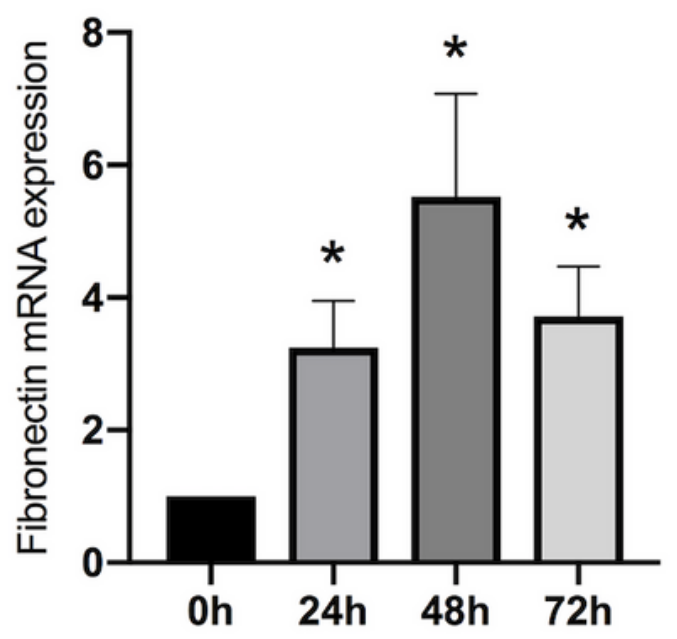

G

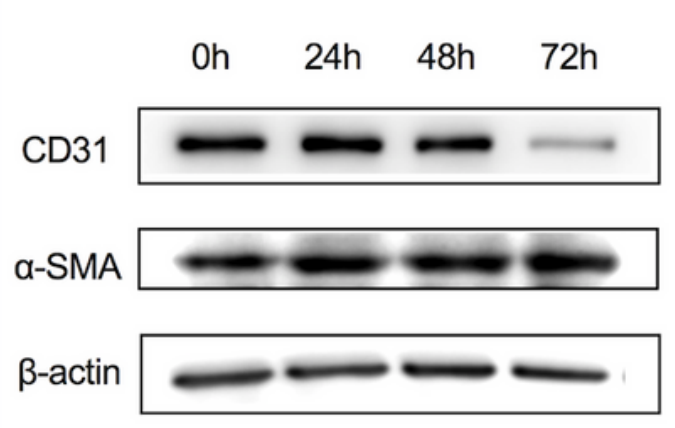

$\mathrm{F}$

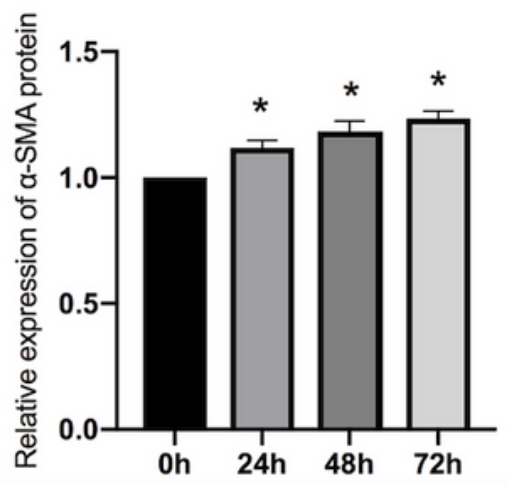

Figure 2

Hypoxia-induced changes in endothelial and fibrotic markers expression. (A,B,C,D) ECs were exposed to hypoxia for $72 \mathrm{~h}$ and mRNA expression was analyzed. (E)Representative images from western blot experiments performed for detection of endothelial marker CD31, and fibrotic marker a-SMA as in $E .(F, G)$ Densitometric analyses of the western blot experiments. Statistical differences were assessed by a one- 
way analysis of variance (ANOVA) (Kruskal-Wallis) followed by Dunn's post hoc test. *: $P<0.05$ against the untreated condition. Graph bars show the mean \pm SD.

A

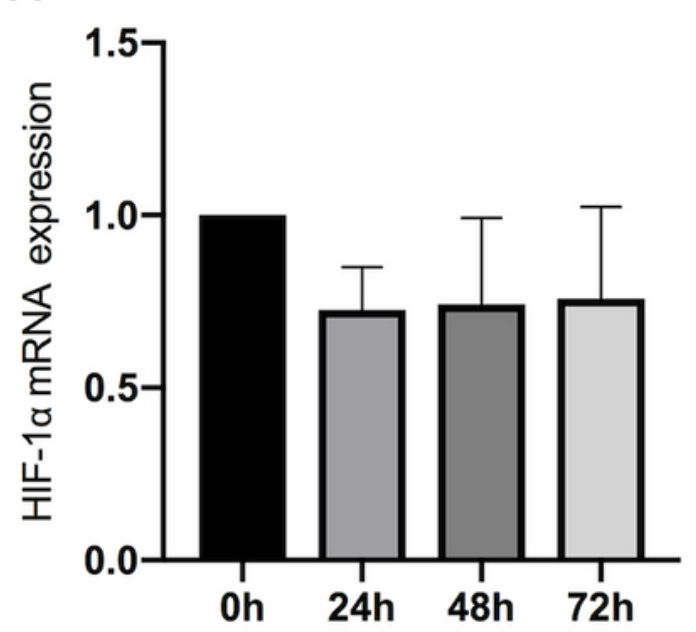

$\mathrm{C}$

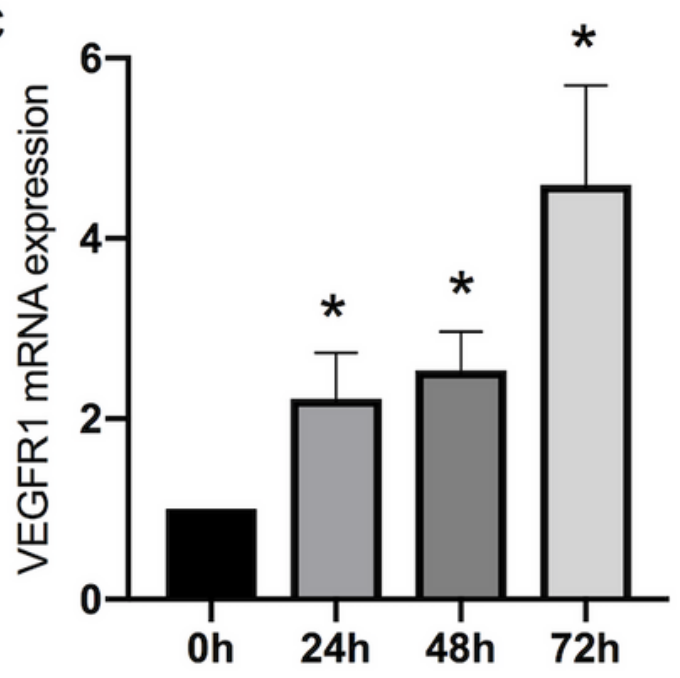

B
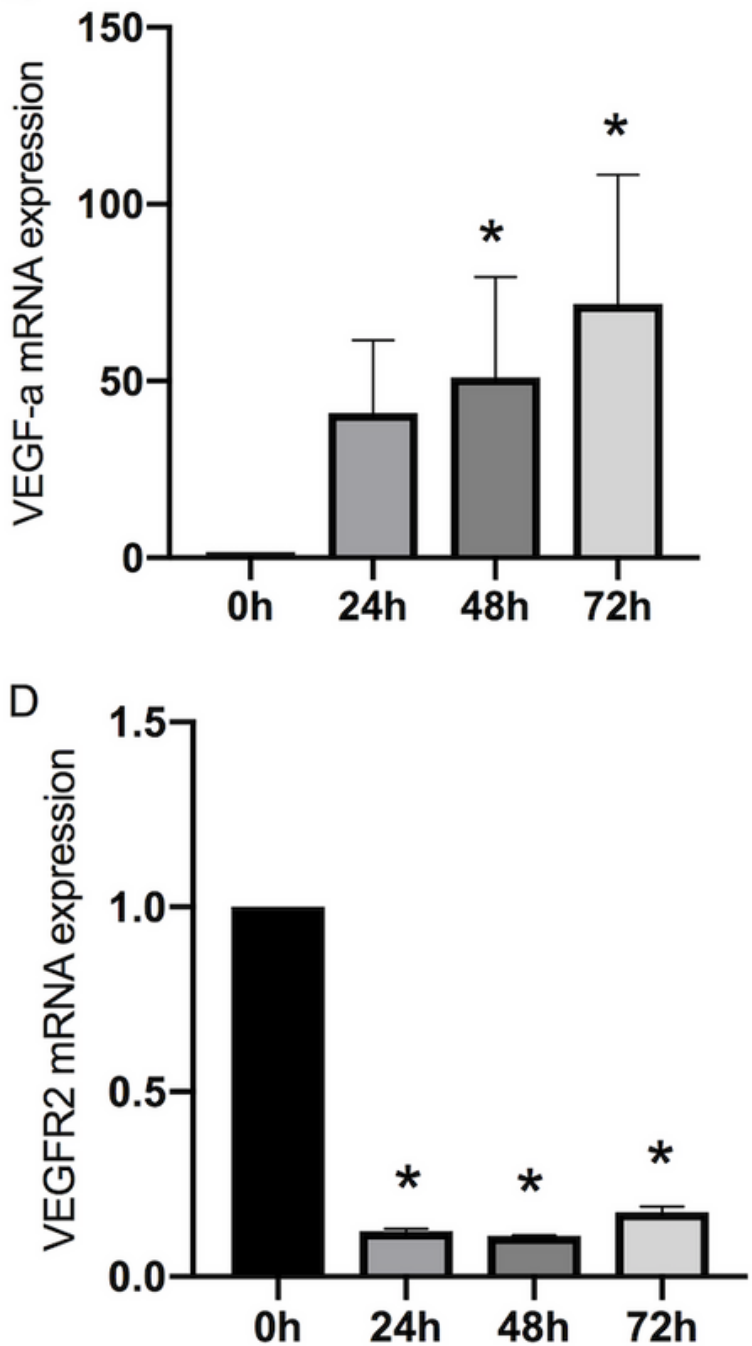

G
E

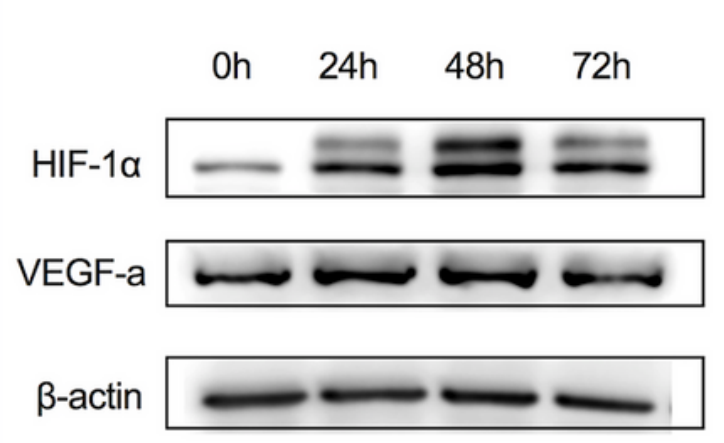

$\mathrm{F}$

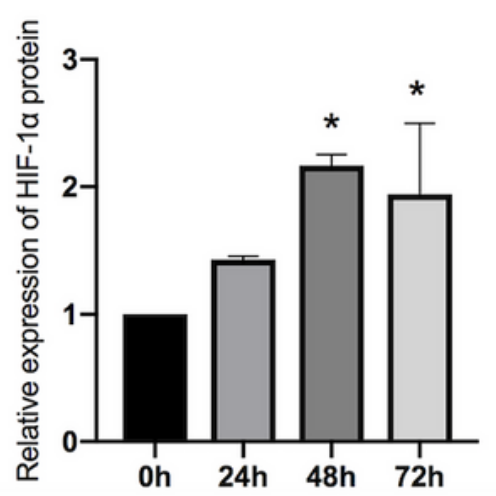

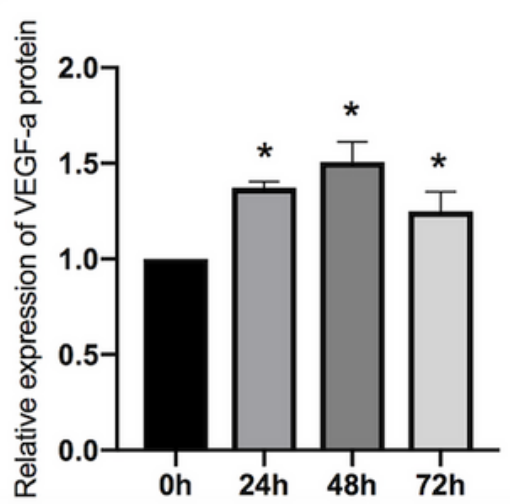

Figure 3

Hypoxia-induced changes of HIF-1a, VEGF-a, VEGFR1, VEGFR2 at mRNA and protein level. (A,B,C,D) ECs were exposed to hypoxia for $72 \mathrm{~h}$ and mRNA expressions of HIF-1a,VEGF-a, VEGFR1, VEGFR2 were analyzed. (E) Representative images from western blot experiments performed for detection of HIF-1a 
and VEGF-a. ( $F$ and G) Densitometric analyses of the western blot experiments. Statistical differences were assessed by a one-way analysis of variance (ANOVA) (Kruskal-Wallis) followed by Dunn's posthoc test. *: $\mathrm{P}<0.05$ against the untreated condition. Graph bars show the mean \pm SD.

A

Oh $24 \mathrm{~h} \quad 48 \mathrm{~h} \quad 72 \mathrm{~h}$

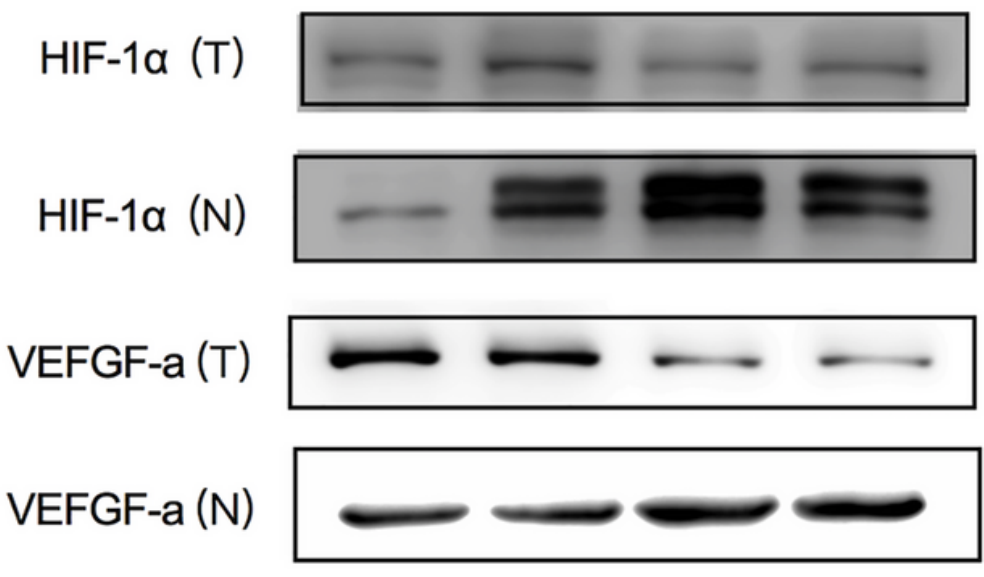

$\beta$-actin

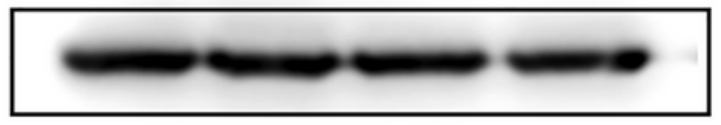

B

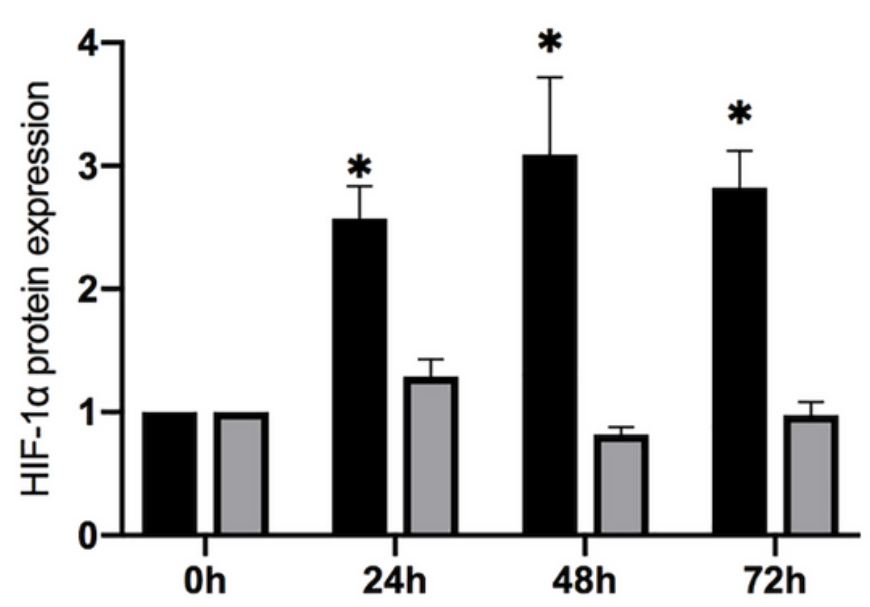

C

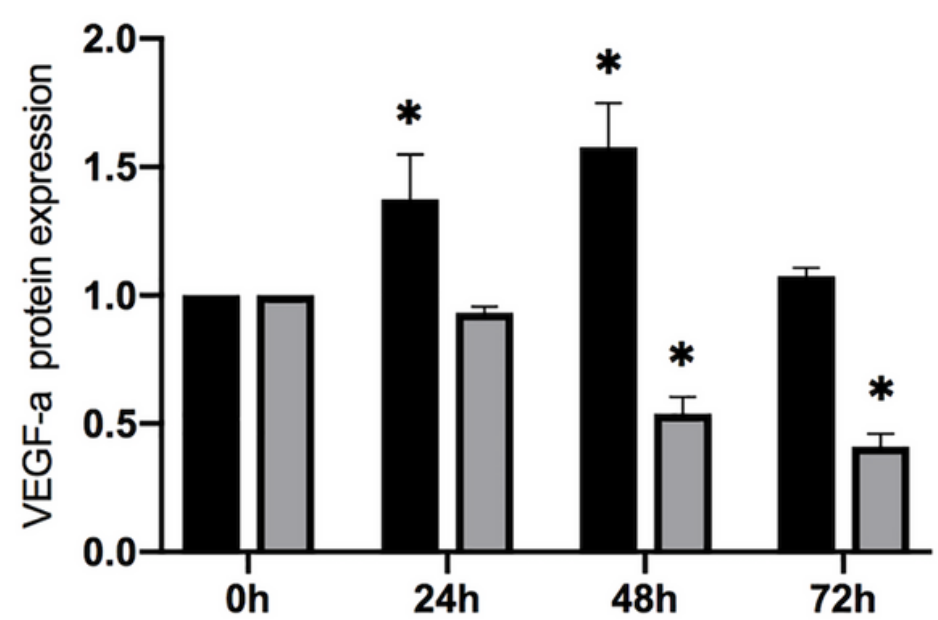

Figure 4

Hypoxia-treated ECs was incubated with tamoxifen,the HIF-1a inhibitor, and the expressions of HIF-1a, VGEF-a were detected. (A) Representative images from western blot experiments performed for detection of HIF-1a, VGEF-a with and without tamoxifen treatment. $(B, C)$ Densitometric analyses of the western blot experiments. Statistical differences were assessed by a one-way analysis of variance (ANOVA) (KruskalWallis) followed by Dunn's posthoc test. *: $\mathrm{P}<0.05$ against the untreated condition. Graph bars show the mean \pm SD. 
A

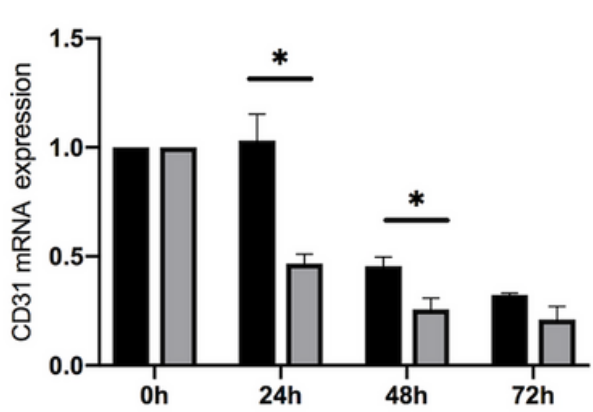

D

Oh $\quad 24 \mathrm{~h} \quad 48 \mathrm{~h} \quad 72 \mathrm{~h}$

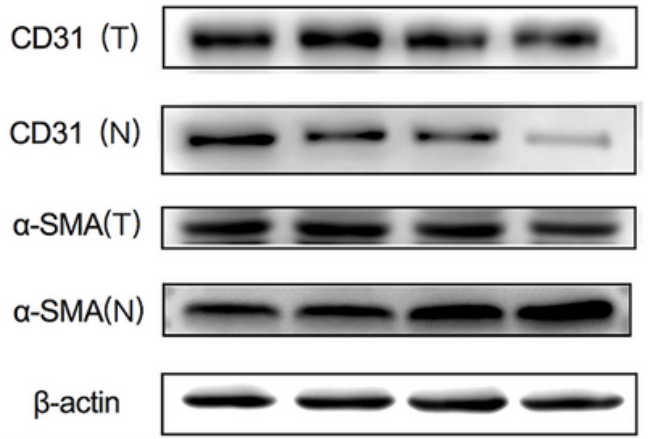

B

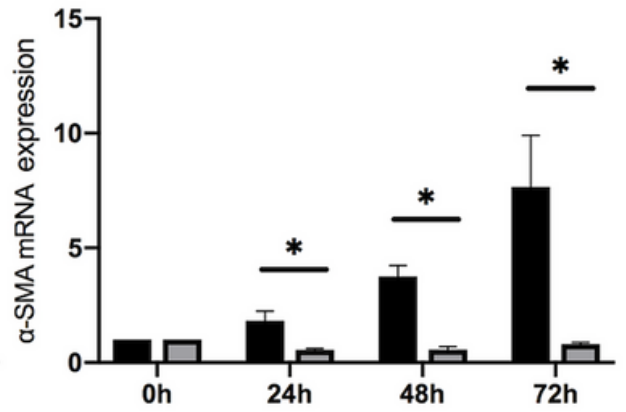

E

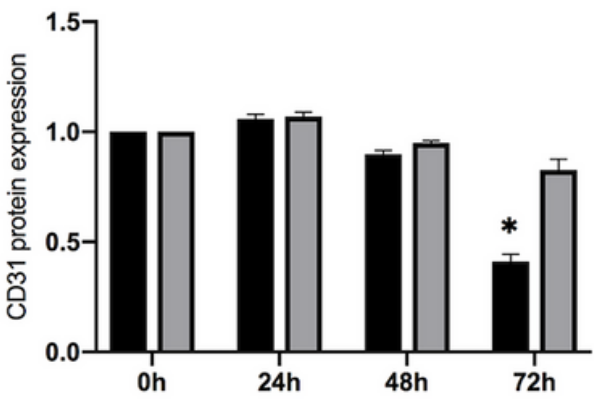

C

שTamoxifen

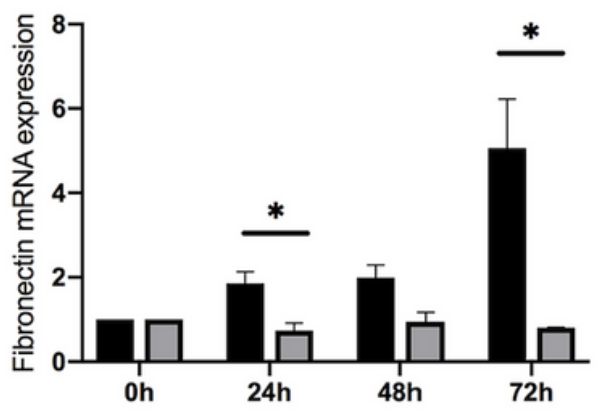

F

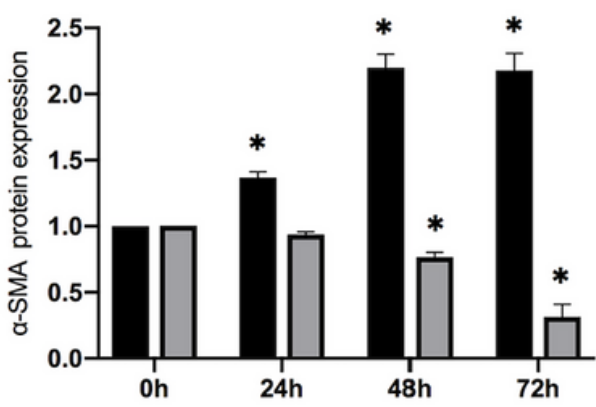

\section{Figure 5}

Hypoxia-treated ECs was incubated with tamoxifen, the HIF-1a inhibitor, and the expressions of CD31, fibronectin and $a-S M A$ were detected. $(A, B, C)$ The relative expression of CD31, fibronectin and a-SMA mRNA. (D) Representative images from western blot experiments performed for detection of HIF-1a, VGEF-a with and without tamoxifen treatment. (E,F) Densitometric analyses of the western blot experiments. Statistical differences were assessed by a one-way analysis of variance (ANOVA) (KruskalWallis) followed by Dunn's posthoc test. *: $\mathrm{P}<0.05$ against the untreated condition. Graph bars show the mean \pm SD. 
A

Oh $\quad 24 \mathrm{~h} \quad 48 \mathrm{~h} \quad 72 \mathrm{~h}$

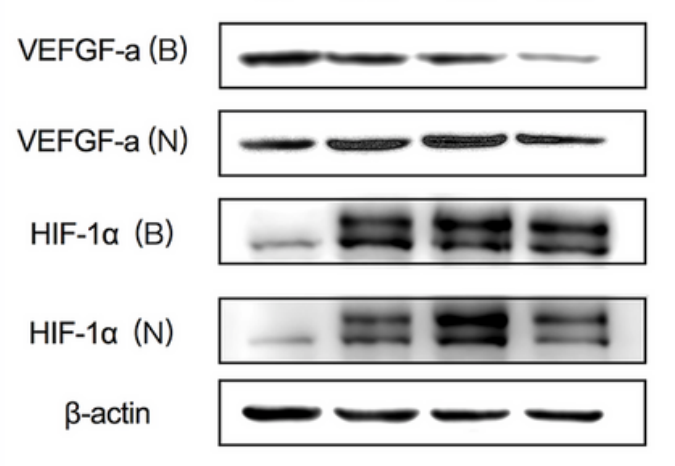

$\begin{array}{ccccc}D & \text { Oh } & 24 \mathrm{~h} & 48 \mathrm{~h} & 72 \mathrm{~h} \\ \mathrm{CD} 31 \text { (B) } & - & - & & \end{array}$

$\mathrm{CD} 31(\mathrm{~N}) \longrightarrow 00$

$\alpha-S M A(B)$

a-SMA(N)

$\beta$-actin
B

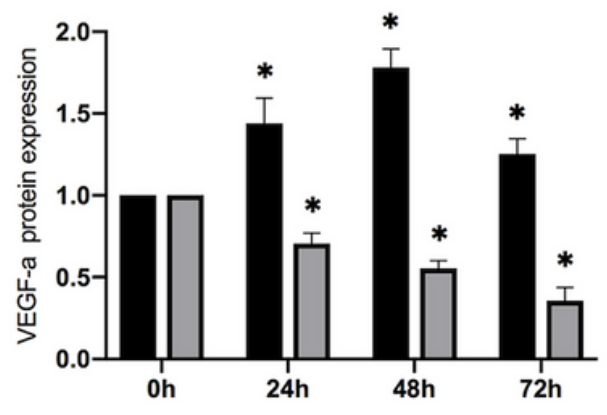

$\mathrm{E}$

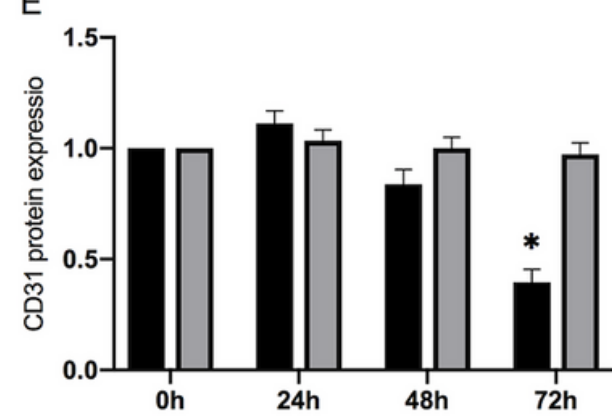

C Control
Bevacizumab

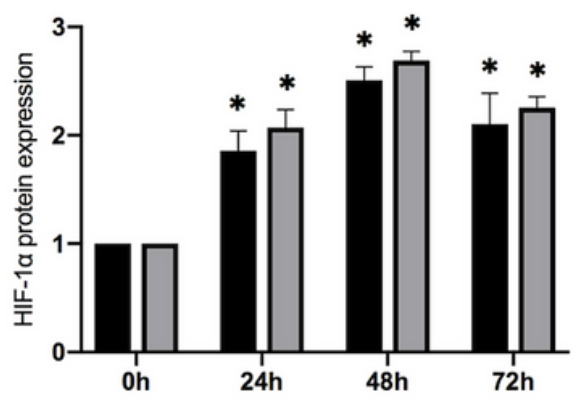

$\mathrm{F}$

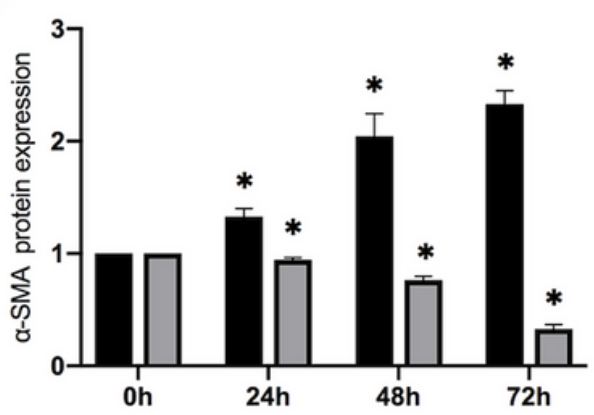

Figure 6

Hypoxia-treated ECs was incubated with bevacizumab, the VGEF-a inhibitor, and the expressions of HIF1a, VGEF-a, CD31, and a-SMA were detected. (A,D) Representative images from western blot experiments performed for detection of HIF-1a, VGEF-a, CD31, and a-SMA with and without bevacizumab treatment. $(B, C, E, F)$ Densitometric analyses of the western blot experiments. Statistical differences were assessed by a one-way analysis of variance (ANOVA) (Kruskal-Wallis) followed by Dunn's posthoc test. *: $P<0.05$ against the untreated condition. Graph bars show the mean \pm SD.

\section{Supplementary Files}

This is a list of supplementary files associated with this preprint. Click to download.

- Additionalfile1.xls

- Additionalfile2.doc 